\title{
Modeling Personality Structure Using Semantic Relationships: Is the HEXACO Honesty-Humility a Distinct Trait?
}

\author{
C. M. Hew D. Gill, Elizaveta Berezina \\ Sunway University, Malaysia \\ *Corresponding author. E-mail: hewg@sunway.edu.my
}

Background. Although the Big Five model (BFM) of personality has been the dominant paradigm in personality research since the mid-1990s, it has recently been challenged by the HEXACO model, which contains an additional factor called Honesty-Humility. Since both these models of personality were developed using the same factor analytic techniques, there has been an ongoing but inconclusive debate about the relative merits of these competing models.

Objective. This paper assesses the robustness of the Honesty-Humility trait using a technique based on the semantic relationships between personality trait adjectives.

Design. Trait marker adjectives for the HEXACO Honesty-Humility and BFM Agreeableness and Neuroticism personality domains in the English language are translated into, and back-translated from, six Asian languages to generate lists of closely related trait terms known as schedonyms. The numbers of schedonyms found within and across the three personality domains are then compared, to determine whether the HEXACO Honesty-Humility factor is semantically distinct from the BFM traits of Agreeableness and Neuroticism.

Results. Our findings indicate that the Honesty-Humility trait domain is semantically distinct from the BFM traits of Agreeableness and Neuroticism, and therefore that there is at least one more personality trait beyond the BFM. The implications of these findings, and the potential applications of this semantically-based technique for establishing the universal structure of the human personality, are briefly discussed.

Conclusion. Our semantic analysis provides clear evidence that there is an Honesty-Humility trait domain in addition to the Agreeableness and Neuroticism traits, and therefore that HEXACO provides a better description of human personality than the BFM.

Keywords: Big Five Model (BFM), HEXACO, HonestyHumility (H6), Agreeableness, Neuroticism, schedonyms, lexical argument, private language argument, Asian languages, personality traits 


\section{Introduction}

Over the past two and a half decades, the "Big Five" model (BFM) of personality traits, which consists of Extraversion, Agreeableness, Conscientiousness, Neuroticism, and Openness to Experience, has gained much support among researchers as a description of the fundamentals of human personality (Borghuis et al., 2017; Hodgkinson \& Gill, 2015; Leutner, Ahmetoglu, Akhtar, \& Chamorro-Premuzic, 2014; Shmelev, 2002). The BFM is said to have a biological basis (Power \& Pluess, 2015; Trofimova, 2016), and to constitute a generalizable description of personality that is applicable across all cultures and languages (Kajonius et al., 2017; Schmitt et al., 2007). However, since its inception, the BFM has had its critics, with some suggesting the BFM may be too complex (e.g. Mitchell \& Kumari, 2016; Gurven, von Rueden, Massenkoff, Kaplan, \& Lero Vie, 2013; Eysenck, 1992), and even apparent advocates for the BFM arguing that it may not be complex enough (Barelds \& De Raad, 2015; Cattell, 1995; Mõttus, Kandler, Bleidorn, Riemann, \& McCrae, 2017; Waller, 1995).

More recently, the six-factor HEXACO model of personality, which adds the trait of Honesty-Humility to the others (Extraversion, Agreeableness, Conscientiousness, Emotional Stability, and Openness) (Ashton, Lee, \& Boies, 2015), has begun to attract some scholarly support (Anglim, Leivens, Everton, Grant, \& Marty, 2018; Strouts, Brase, \& Dillon, 2017; Shu, McAbee, \& Ayman, 2017).

Since the BFM and HEXACO were primarily developed by using factor analysis ${ }^{1}$ of personality trait adjectives, much of the debate about which model is best has hinged on the various methodological decisions made by the researchers conducting the analyses. However, there are no universally agreed-upon and objective criteria for carrying out a factor analysis. Therefore, selecting the number of factors to extract, and choosing the method of factor rotation, are matters decided by each individual researcher; multiple related models can be extracted from the same dataset (Loehlin, 2013). Unsurprisingly, the lack of a common approach has led to considerable disagreement about which technique(s) may or may not be correct, the number of factors that should be extracted, and even the relatedness or orthogonality of those factors (Davies, Connelly, Ones, \& Birkland, 2015; de Vries, 2011; Putilov, 2018; Wright, 2017). This, in turn, has meant that the validity of any particular personality model has become, for many, a matter of personal judgment, and led some to call for a paradigm shift in our approach to the measurement of personality (Uher, 2013).

The BFM and HEXACO were both developed within the lexical hypothesis (Galton, 1884; Saucier, \& Goldberg, 1996), which proposes that important personality characteristics will be encoded in a language as a single word (De Raad \& Mlačić, 2017; John, Angleitner, \& Ostendorf, 1988). The factor analytic approach relies on the assumption that when participants use personality trait adjectives to rate themselves, the ratings will show some correlations because many trait adjec-

1 Actually, most personality researchers have used principal components analysis (PCA) instead of factor analysis, but since the latter term has become so embedded in the literature, it shall be used here. It should be noted that, although PCA is an approach that falls within the broad family of factor analytic techniques, it is subject to a set of assumptions and constraints that differ in some significant respects from general factor analysis; by far the most accessible introduction to the field is Paul Kline's $(1993 ; 2014)$ An Easy Guide to Factor Analysis. London: Routledge. 
tives will be synonyms-i.e., descriptions of closely related aspects of personality which are semantically and psychologically related (Saucier \& Goldberg, 1996).

However, the lexical argument pre-dates Wittgenstein's $(1953 ; 2009)$ private language argument (PLA), which asserts that language can only exist in the public domain where semantic meanings are agreed upon between language users (Leontiev, 2017; Vygotsky, 1987). If we accept that any trait adjective is a publicly agreedupon term for an aspect of personality, then why would language users generate many words that were repetitious synonyms? Personality corpora are extensive and contain many distinct trait adjectives (e.g. Chandler, 2018; Litvinova \& Ryzhkova, 2018), so surely it is more logical to conclude that each trait adjective describes a unique aspect of the personality, and although it may be true that many trait adjectives are related, they are not exact synonyms, but rather schedonyms - descriptive terms that are close but not identical in meaning.

So, for example, trait adjectives that are often considered to be synonyms-such as friendly, gregarious, likeable, outgoing, pleasant, and sociable-are not actually terms that can be used interchangeably. They are in fact schedonyms, which only exist as trait adjectives because they make meaningful and publicly agreed-upon distinctions among different aspects of human personality. Thus, any trait domain should contain groups of schedonyms, some of which are very close in meaning, and some of which may not be particularly close in meaning.

For example, people who are friendly may also be sociable, outgoing, and pleasant, but it is not necessarily the case that people who are outgoing are always pleasant or friendly. This is why these distinct trait adjectives exist as schedonyms, which are closely related but not semantically identical. Similarly, the trait adjectives $d u t i$ ful, honest, honorable, and responsible may be close in meaning to each other, but they are not semantically identical, and they are clearly semantically distant from the trait adjectives sociable, outgoing, and pleasant.

Clusters of schedonyms have developed over thousands of years, as millions of language users have developed agreed-upon meanings to describe close but distinct aspects of human personality. Even though human languages have progressively differentiated from their common origins (Velichkovsky \& Rumbaugh, 2013), if the structure of the human personality is universal, then the trait adjectives found in one language should have analogues in other languages, and these trait adjectives should be related in broadly consistent ways. This means that when a trait adjective is translated from a first or meta-language, into a second target language, and then back-translated to the meta-language, the result should be the same trait adjective in the original meta-language, and a cluster of its closest related trait adjective schedonyms.

Schedonyms act as semantic tags, with their clusters showing close semantic, and hence psychological, relationships. Conversely, trait adjectives from distinct personality trait domains should share very few, or even zero, schedonyms. So, for example, when the target trait adjective friendly is translated and back-translated, it should yield the adjective friendly, and might also yield schedonyms such as gregarious, likeable, outgoing, pleasant, and sociable in nearly all languages, but the list of schedonyms might not be identical across different languages. Then, when the trait adjective outgoing is translated and back-translated, it might yield the schedonyms gregarious and sociable in all languages, and the adjectives likeable and pleasant 
in some languages, while in a few languages, it might yield demonstrative and expansive. When expansive is back-translated, it might yield generous and liberal in all languages, with outgoing and friendly in some languages.

By translating and back-translating trait adjectives through multiple languages, it should be possible to assess the relatedness of trait adjectives, and the strength of their semantic and psychological relationships, based upon the number of schedonyms shared between them. This means that counting the number of schedonyms shared between individual trait adjectives could offer a viable alternative to factoranalysis as a means of assessing the content and robustness of personality domains.

The HEXACO model is effectively composed of the BFM plus the HonestyHumility trait (H6), which is derived using adjectives (and therefore factor-analytic variance) that other researchers would consider to be part of the BFM Agreeableness and Neuroticism trait domains (Ashton, Lee, \& de Vries, 2014). If H6 is independent of the Agreeableness and Neuroticism domains, then it would be expected that $\mathrm{H} 6$ trait marker adjectives would share very few or no schedonyms with trait marker adjectives from the two BFM traits. The present study will use schedonyms to investigate the extent to which the putative $\mathrm{H} 6$ trait domain is related to, or distinct from, the BFM's Agreeableness and Neuroticism traits.

\section{Method}

Using the trait adjective lists provided by Saucier \& Goldberg (1996) and Ashton et al. (2015), we identified 10 marker adjectives for each of the trait domains for the BFM's Agreeableness and Neuroticism, and HEXACO's H6, as shown in Table 1.

Table 1

Trait marker adjectives representative of H6, Agreeableness, and Neuroticism

\begin{tabular}{ccc}
\hline $\begin{array}{c}\text { H6 } \\
\text { Trait Adjectives from } \\
\text { Ashton et al. (2015) }\end{array}$ & $\begin{array}{c}\text { Agreeableness } \\
\text { Trait Adjectives from } \\
\text { Saucier \& Goldberg (1996) }\end{array}$ & $\begin{array}{c}\text { Neuroticism } \\
\text { Trait Adjectives from } \\
\text { Saucier \& Goldberg (1996) }\end{array}$ \\
\hline sincere & sympathetic & moody \\
generous & kind $^{*}$ & touchy \\
honest & cooperative & temperamental \\
trustworthy & courteous & irritable \\
loyal & compassionate & emotional \\
snobbish & harsh & relaxed \\
greedy & rude & patient \\
deceitful & antagonistic & brave \\
conceited & abusive & casual \\
superficial & egotistical & earthy \\
\hline
\end{tabular}

${ }^{*}$ Note: the trait adjectives kind and compassionate were also listed by Ashton et al. (2015) as H6 trait terms, but as this would have immediately meant a perfect match between $20 \%$ of the available trait adjectives, it was decided to select alternative adjectives as trait markers. 
The initial list did not include any privative constructed negatives - words that use the English prefixes in/un/dis to form negatives (e.g. insincere, unkind) - because 1) these terms are not independently generated trait adjectives, and 2) when they are translated into target languages, they often yield phrases beginning with He/tidak/不/wala, etc. Similarly, compound terms using a hyphen to connect two ideas (e.g. self-centered; warm-hearted) were not selected, because similar compound terms might not exist in the same form in some languages, and they could also be translated as idiomatic phrases unique to the language in question.

Since the H6 trait has been found in several factor analytic studies conducted in Asia (De Raad \& Mlacic, 2017; Fan, Zhichen, Beibei, \& Jixia, 2015; Han, Seok, \& Kim, 2017; Kawamoto, 2016), the present study used English as the first or metalanguage, with six South East Asian languages from three language families as target languages, to yield a broad spread of trait adjectives from back-translation, and to boost generalizability.

Table 2

Target languages and language families used for translating the trait marker adjectives

\begin{tabular}{llllll}
\hline \multicolumn{2}{c}{ Austronesian } & \multicolumn{2}{c}{$\begin{array}{c}\text { Austroasiatic } \\
\text { (Mon-Khmer) }\end{array}$} & \multicolumn{2}{c}{$\begin{array}{c}\text { Tai-Kadai } \\
\text { (Zhuang-Tai) }\end{array}$} \\
\hline Indonesian & Filipino & Khmer & Vietnamese & Lao & Thai \\
\hline
\end{tabular}

The initial trait adjectives were translated from English into the target languages using 20 public domain bilingual dictionaries; then the resulting adjectives were back-translated into English to generate lists of schedonyms for each of the trait marker adjectives. Again, any compound terms that were yielded during translation (e.g. home-felt, pure-minded, fair-spoken) were eliminated. As an example, the trait adjective sincere, when translated into Bahasa Indonesia, yielded the adjective tulus, which, when back-translated, yielded sincere and 12 more adjectives, including candid, honest, truthful, and upright; nine compound terms, including heartwhole, single-eyed, and true-blue, were excluded from further analysis. When honest was translated into Thai, it yielded the term sụ̂xs̄aty (ซื่อสัตย์), that, when backtranslated, yielded honest and 40 more adjectives, of which four were excluded and 36 retained, including candid, sincere, truthful, and upright.

\section{Calculation of Intra-trait and Inter-trait Schedonyms}

Following from the above, we can see that within the putative H6 personality domain, the initial trait adjective sincere, when translated into and back from Bahasa Indonesia, yielded the four adjectives candid, honest, truthful, and upright, and that the Thai translation of honest yielded candid, sincere, truthful, and upright. Each of these terms was therefore counted as one intra-trait schedonym within H6 for each initial trait adjective, making a total of four per initial adjective, and eight in total. However, note that the direct back-translations of sincere to sincere in Bahasa Indonesia, and honest to honest in Thai, were not counted as intra-trait schedonyms, and that no direct back-translations of the initial adjective terms were counted for any 
of the target languages, as this would have artificially inflated the apparent number of intra-trait schedonyms.

The inter-trait schedonyms shared between the personality trait domains were counted in the same way. The back-translations of the $\mathrm{H} 6$ initial adjective generous in Thai, and the Agreeableness initial adjective kind in Filipino, both yielded considerate, and this was counted as one schedonym for each initial trait adjective, for a total count of two inter-trait schedonyms. When an $\mathrm{H} 6$ initial adjective yielded a schedonym the same as an Agreeableness initial adjective, or vice versa, the schedonym was counted as an inter-trait one, to capture the relationship between initial trait adjectives from different personality domains.

\section{Results and Discussion}

The numbers of trait adjectives generated following the translation and back-translation of the initial trait marker adjectives are shown in Tables 3, 4 and 5.

Table 3

Number of schedonyms generated for $\mathrm{H} 6$ trait adjectives by translation into and backtranslation from the six Asian languages

\begin{tabular}{cccccccc}
\hline $\begin{array}{c}\text { H6 } \\
\text { Initial Trait } \\
\text { Adjectives }\end{array}$ & Indonesian & Filipino & Khmer & Vietnamese & Lao & Thai & Totals \\
\hline sincere & 58 & 25 & 40 & 24 & 37 & 72 & 256 \\
generous & 18 & 14 & 15 & 11 & 11 & 33 & 102 \\
honest & 73 & 127 & 58 & 28 & 37 & 121 & 444 \\
trustworthy & 31 & 25 & 67 & 18 & 15 & 34 & 190 \\
loyal & 36 & 132 & 36 & 30 & 40 & 59 & 333 \\
snobbish & 12 & 10 & 17 & 1 & 20 & 8 & 68 \\
greedy & 5 & 3 & 3 & 8 & 14 & 12 & 45 \\
deceitful & 24 & 21 & 22 & 18 & 9 & 24 & 118 \\
conceited & 51 & 28 & 28 & 39 & 21 & 39 & 206 \\
superficial & 6 & 6 & 8 & 3 & 0 & 15 & 38 \\
\hline TOTALS & 314 & 391 & 294 & 180 & 204 & 417 & 1800 \\
\hline
\end{tabular}

The $\mathrm{H} 6$ adjectives yielded a reasonable number of adjectives, with Bahasa Indonesia, Filipino, and Thai having the largest number of schedonyms, although it should be noted that Lao yielded comparatively few schedonyms because few dictionary resources were available. The trait adjective with the largest number of schedonyms was honest, followed by loyal and sincere, which might be considered as anchor trait adjectives for the Honesty pole of the H6 personality domain. 
Table 4

Number of schedonyms generated for Agreeableness trait adjectives by translation into and back-translation from the six Asian languages

\begin{tabular}{cccccccc}
\hline $\begin{array}{c}\text { Agreeableness } \\
\text { Initial Trait } \\
\text { Adjectives }\end{array}$ & Indonesian & Filipino & Khmer & Vietnamese & Lao & Thai & Totals \\
\hline sympathetic & 24 & 21 & 7 & 19 & 6 & 37 & 114 \\
kind & 76 & 86 & 53 & 19 & 18 & 67 & 319 \\
cooperative & 2 & 12 & 1 & 2 & 1 & 1 & 19 \\
courteous & 83 & 42 & 13 & 55 & 12 & 61 & 266 \\
compassionate & 36 & 31 & 9 & 19 & 7 & 83 & 185 \\
harsh & 46 & 39 & 21 & 53 & 7 & 49 & 215 \\
rude & 120 & 72 & 22 & 67 & 16 & 90 & 387 \\
antagonistic & 0 & 1 & 1 & 1 & 1 & 2 & 6 \\
abusive & 42 & 15 & 9 & 48 & 9 & 105 & 228 \\
egotistical & 6 & 4 & 4 & 4 & 0 & 10 & 28 \\
\hline TOTALS & 435 & 323 & 140 & 287 & 77 & 505 & 1767 \\
\hline
\end{tabular}

Table 5

Number of schedonyms generated for Neuroticism trait adjectives by translation into and back-translation from the six Asian languages

\begin{tabular}{cccccccc}
\hline $\begin{array}{c}\text { Neuroticism } \\
\text { Initial Trait } \\
\text { Adjectives }\end{array}$ & Indonesian & Filipino & Khmer & Vietnamese & Lao & Thai & Totals \\
\hline moody & 28 & 16 & 7 & 24 & 5 & 27 & 107 \\
touchy & 37 & 24 & 0 & 23 & 1 & 4 & 89 \\
temperamental & 4 & 15 & 3 & 9 & 3 & 27 & 61 \\
irritable & 50 & 36 & 2 & 25 & 9 & 18 & 140 \\
emotional & 10 & 11 & 3 & 10 & 3 & 3 & 40 \\
relaxed & 6 & 1 & 1 & 3 & 0 & 7 & 18 \\
patient & 7 & 3 & 0 & 7 & 2 & 6 & 25 \\
brave & 42 & 45 & 22 & 25 & 1 & 42 & 177 \\
casual & 16 & 3 & 6 & 7 & 3 & 5 & 40 \\
earthy & 46 & 11 & 22 & 1 & 5 & 49 & 134 \\
\hline TOTALS & 246 & 165 & 66 & 134 & 32 & 188 & 831 \\
\hline
\end{tabular}


Agreeableness yielded fewer schedonyms than H6, but with Bahasa Indonesia, Filipino, and Thai again yielding the most schedonyms. The adjective rude had most schedonyms, followed by kind, but the adjectives antagonistic and egotistical generated relatively few back-translations. This may have been because antagonistic and egotistical are relatively uncommon words in English so that few translations have been yet been generated in the target languages. The trait adjective cooperative also generated few schedonyms, and this was partially because the term was commonly translated as a noun meaning "farm co-operative" in several of the target languages.

The Neuroticism trait adjectives had the fewest schedonyms, although again, the proportions of schedonyms from each target language were broadly consistent with those for the other two personality domains. The trait adjectives with the greatest number of schedonyms were earthy and brave, but, in comparison, there were few schedonyms for temperamental, emotional, and relaxed.

\section{Comparison of Intra-trait vs Inter-trait Schedonyms}

As explained above, trait adjectives from within the same trait should have a relatively high count of intra-trait schedonyms, and more intra-trait schedonyms than inter-trait schedonyms shared with unrelated trait domains. Thus, the total number of intra-trait schedonyms should be significantly higher than the number of inter-trait schedonyms, and this appeared to be the case when the intratrait and inter-trait schedonym counts were compared as shown in Tables 6, 7, and 8 below.

Table 6

Number of intra-trait and inter-trait schedonyms for the H6 trait adjectives across the six Asian languages

\begin{tabular}{cccc}
\hline $\begin{array}{c}\text { H6 } \\
\text { Trait Adjectives }\end{array}$ & $\begin{array}{c}\text { H6 } \\
\text { intra-trait schedonyms }\end{array}$ & $\begin{array}{c}\text { Agreeableness inter- } \\
\text { trait schedonyms }\end{array}$ & $\begin{array}{c}\text { Neuroticism inter-trait } \\
\text { schedonyms }\end{array}$ \\
\hline sincere & 256 & 13 & 1 \\
Generous & 102 & 225 & 11 \\
Honest & 444 & 53 & 5 \\
trustworthy & 190 & 12 & 0 \\
loyal & 333 & 12 & 1 \\
snobbish & 68 & 24 & 1 \\
greedy & 45 & 8 & 0 \\
deceitful & 118 & 3 & 2 \\
conceited & 206 & 64 & 0 \\
superficial & 38 & 5 & 4 \\
\hline TOTALS & 1800 & 419 & 25 \\
\hline
\end{tabular}


The figures in Table 6 show that the H6 positive-pole trait adjectives sincere, honest, trustworthy, and loyal produced a relatively large number of intra-trait schedonyms but few inter-trait schedonyms, exactly the pattern which would be expected if these terms are at the core of a distinct personality domain. Conversely, generous yielded a comparatively lower number of intra-trait schedonyms, but higher numbers of inter-trait schedonyms with the Agreeableness domain, suggesting that it might be drawn from the Agreeableness rather than the H6 personality domain.

Among the $\mathrm{H} 6$ negative-pole adjectives, snobbish and conceited had a moderate number of intra-trait schedonyms, but greedy, deceitful, and superficial had very few schedonyms within the H6 domain, or across the other two domains. This suggests that the negative pole of H6 is not particularly well-defined by these five trait adjectives. Indeed, since deceitful is an antonym for sincere, honest, and trustworthy, yet shares few schedonyms with the other four adjectives, it seems probable that snobbish, conceited, greedy, and superficial do not capture the negative pole of the Honesty aspect of H6. However, the fact that snobbish and conceited share a fair number intra-trait schedonyms, yet are unrelated to Agreeableness or Neuroticism, suggests they may capture the negative pole of the Humility aspect of H6.

Table 7

Number of intra-trait and inter-trait schedonyms for the Agreeableness trait adjectives across the six Asian languages

\begin{tabular}{cccc}
\hline $\begin{array}{c}\text { Agreeableness } \\
\text { Trait Adjectives }\end{array}$ & $\begin{array}{c}\text { Agreeableness } \\
\text { intra-trait schedonyms }\end{array}$ & $\begin{array}{c}\text { H6 } \\
\text { inter-trait schedonyms }\end{array}$ & $\begin{array}{c}\text { Neuroticism inter- } \\
\text { trait schedonyms }\end{array}$ \\
\hline sympathetic & 114 & 43 & 5 \\
kind & 319 & 25 & 3 \\
cooperative & 19 & 0 & 0 \\
courteous & 266 & 14 & 19 \\
compassionate & 185 & 4 & 4 \\
harsh & 215 & 7 & 109 \\
rude & 387 & 14 & 263 \\
antagonistic & 6 & 0 & 0 \\
abusive & 228 & 6 & 109 \\
egotistical & 28 & 79 & 0 \\
\hline TOTALS & 1767 & 192 & 512 \\
\hline
\end{tabular}

The Agreeableness adjectives (See Table 7) had many intra-trait schedonyms, which suggested a high degree of semantic relatedness, and that most of the adjectives came from the same personality domain. The Agreeableness antonyms antagonistic and egotistical shared few schedonyms with other Agreeableness trait 
adjectives, and egotistical appeared to be more closely related to the H6 domain, possibly because it may be part of the negative pole of the Humility aspect of $\mathrm{H} 6$.

Table 8

Number of intra-trait and inter-trait schedonyms for the Neuroticism trait adjectives across the six Asian languages

\begin{tabular}{lccc}
\hline $\begin{array}{c}\text { Neuroticism } \\
\text { Trait Adjectives }\end{array}$ & $\begin{array}{c}\text { Neuroticism } \\
\text { intra-trait schedonyms }\end{array}$ & $\begin{array}{c}\text { H6 } \\
\text { inter-trait schedonyms }\end{array}$ & $\begin{array}{c}\text { Agreeableness inter- } \\
\text { trait schedonyms }\end{array}$ \\
\hline moody & 107 & 0 & 28 \\
touchy & 89 & 3 & 25 \\
temperamental & 61 & 0 & 14 \\
irritable & 140 & 1 & 38 \\
emotional & 40 & 0 & 7 \\
relaxed & 18 & 0 & 1 \\
patient & 25 & 8 & 15 \\
brave & 177 & 3 & 42 \\
casual & 40 & 5 & 29 \\
earthy & 134 & 5 & 342 \\
\hline TOTALS & 831 & 25 & 541 \\
\hline
\end{tabular}

The Neuroticism trait adjectives yielded fewer intra-trait schedonyms than the other domains, although moody, touchy, temperamental, and irritable did show some modest counts and ratios. There were comparatively few schedonyms at the Emotional Stability pole of this domain, with the adjectives patient and relaxed having very few intra-trait schedonyms, despite the fact that they are generally associated with the positive pole of this domain. This may have been because the trait adjectives brave, casual, and earthy are actually drawn from other domains; for example, brave has been identified in other studies as part of the positive pole of Extraversion, and casual as part of the negative pole of Conscientiousness (e.g. Gill \& Hodgkinson, 2007). The figures in Table 8 suggest that earthy may be more closely associated with the Agreeableness domain.

The figures presented in Tables 6, 7, and 8 showed that many of the trait marker adjectives in each of the three personality domains had more intra-trait schedonyms than inter-trait schedonyms, suggesting that H6, Agreeableness, and Neuroticism are semantically distinct. In order to investigate whether or not these different patterns of semantic relationships were significantly different, the numbers of intra-trait and inter-trait schedonyms were compared across the three trait domains using the Kruskal-Wallis test.

There is a significant difference in the number of intra-trait schedonyms shared within the $\mathrm{H} 6$ domain, as compared with the number of inter-trait schedonyms shared between the H6 domain and the Agreeableness and Neuroticism domains; 
$(\mathrm{H}(2)=21.83, \mathrm{p}<0.01)$ with a mean rank of 24.40 for H6 intra-trait schedonyms, a mean rank of 16.05 for inter-trait schedonyms between H6 and Agreeableness, and a mean rank of 6.04 for inter-trait schedonyms between H6 and Neuroticism.

There is also a significant difference in the number of intra-trait schedonyms shared within the Agreeableness domain, compared with the number of inter-trait schedonyms shared between the Agreeableness domain, and the H6 and Neuroticism domains; $(\mathrm{H}(2)=10.66, \mathrm{p}<0.01)$ with a mean rank of 22.90 intra-trait schedonyms for Agreeableness, a mean rank of 11.90 for inter-trait schedonyms between Agreeableness and H6, and a mean rank of 11.70 for inter-trait schedonyms between Agreeableness and Neuroticism.

Lastly, there are significant differences in the number of intra-trait schedonyms shared within the Neuroticism domain, compared with the numbers of inter-trait schedonyms shared between the Neuroticism domain and the H6 and Agreeableness domains $(\mathrm{H}(2)=19.59, \mathrm{p}<0.01)$ with a mean rank of 23.35 for Neuroticism intra-trait schedonyms, a mean rank of 6.15 for inter-trait schedonyms between Neuroticism and H6, and a mean rank of 17.00 for inter-trait schedonyms between Neuroticism and Agreeableness.

Overall, these findings show that the H6, Agreeableness, and Neuroticism trait marker adjectives have significantly more semantic relationships within their respective personality domains than they share across other personality domains, suggesting that these traits are robust and semantically distinct personality trait domains.

\section{General Discussion}

The present research was a pilot study designed to investigate the semantic robustness of the H6 personality trait proposed by Ashton et al. (2015), which used a novel approach that could serve as an alternative to factor-analysis, and a means of confirming personality domains using semantic relationships that have evolved over millenia.

The results showed that $\mathrm{H} 6$ is semantically robust and semantically distinct from Neuroticism and Agreeableness, allowing us to tentatively conclude that the HEXACO model of personality, including the H6 Honesty-Humility factor, offers a more comprehensive description of the human personality than the five traits of the BFM. Furthermore, the fact that these semantic relationships were established using trait adjective schedonyms from six South East Asian languages and three major language groups, suggests that the findings of the present study may be generalizable, and that the H6 Honesty-Humility trait domain is likely to be semantically robust in other languages.

However, we should also note that the H6 marker adjectives selected for the present study are mostly representative of the $\mathrm{H} 6$ positive Honesty pole and negative Humility pole. Future research needs to include more adjectives representative of the negative Honesty and positive Humility poles, in order to demonstrate that $\mathrm{H} 6$ is indeed semantically distinct, and also that $\mathrm{H} 6$ is itself a unitary personality domain.

Although it was apparent that some of the trait adjectives within each personality domain had relatively few semantic relationships with other adjectives in the 
same assumed domain, this may have been because the comparatively small number of marker trait adjectives used meant that the full breadth of semantic relationships was not sampled. However, it is also possible that some of the initial trait marker adjectives were not actually drawn from the trait domains to which they were factor-analytically allocated, and instead rightly belong in other personality domains; the most obvious examples were the adjectives brave and casual. Indeed, if brave and casual are actually trait markers for Extraversion and Conscientiousness, then this would explain why they shared so few schedonyms with any of the three factors being investigated in the present study.

Any personality domain should be semantically defined both by the trait adjectives it includes, and those it does not, so ultimately it may be possible to apply this semantically-based technique to much larger samples of trait adjectives. This would allow us to establish the robustness and semantic integrity of any given personality trait domain, as well as the number of personality domains that are present in any collection of trait adjectives. Such an approach might also make it possible to adopt an iterative approach to personality domain specification, by assessing how well individual trait adjectives fit into different domains using the comparative number of intra-trait and inter-trait adjective schedonyms, although clearly this would require much larger initial item pools in order to achieve the statistical power necessary to allocate adjectives accurately.

\section{Limitations}

We must acknowledge that the present study used a limited sample of core trait adjectives drawn from what might be considered "master-lists" for the BFM and HEXACO. This means that further verification is required using a larger pool of trait adjectives. Furthermore, despite the fact that Ashton et al. (2015) identify Agreeableness and Neuroticism as the hierarchical "parents" of H6 Honesty-Humility, it is possible that H6 may not be semantically distinct from Extraversion, Conscientiousness, and Openness. Thus a future study including trait adjectives from all the BFM personality domains alongside H6 Honesty-Humility is clearly required. This may also serve to establish whether brave and casual are part of the Neuroticism trait or other domains, as well as helping to locate the appropriate personality domains for the other trait adjectives which had low numbers of intra-trait and inter-trait schedonyms in the present study.

Another objective of this research was to try out a novel alternative to factoranalysis for assessing the validity of personality trait domains by using semantic relationships. The findings reported above lend some credence to the contention that using translations and back-translations of trait adjectives to generate lists of semantically close personality terms, or schedonyms, may make it possible to map personality trait domains and the boundaries between them using the lexical relationships and distinctions that have evolved in natural language.

Even though the present study was modest in scale, and the techniques applied could be further refined by increasing the initial trait adjective pool, the languages used for translation, and the number of sources used for translation, the logic of the approach seems sound. Sampling more trait adjectives across more languages would increase the number of possible comparisons between groups of trait adjec- 
tives, and the power of the statistical analyses that could be conducted. This would then allow iterative studies to allocate trait adjectives to the correct personality domains, thus making truly valid global comparisons which will help us establish the shape of the universal domains of human personality, as well as those aspects of personality that may be culturally specific. Establishing which are the universal domains, and which the cultural specificities of human personality, must surely be the ultimate goal of personality research, and a technique based on semantic relationships may overcome some of the limitations of factor-analysis to independently validate or challenge the BFM.

\section{Conclusion}

In conclusion, this brief paper suggests that the H6 Honesty-Humility domain is semantically distinct from Agreeableness and Neuroticism, and therefore that HEXACO provides a better description of the human personality than the BFM. Hopefully, by proposing a new semantically based technique for establishing the structure of human personality, this paper represents both a minor milestone, and a modest map, for the greater journey of discovery ahead.

\section{References}

Anglim, J., Lievens, F., Everton, L., Grant, S.L., \& Marty, A. (2018). HEXACO Personality Predicts Counterproductive Work Behavior and Organizational Citizenship Behavior in LowStakes and Job Applicant Contexts. Journal of Research in Personality.

Ashton, M.C., Lee, K., \& Boies, K. (2015). One- through six-component solutions from ratings on familiar English personality-descriptive adjectives. Journal of Individual Differences, 36(3), 183-189. https://doi.org/10.1027/1614-0001/a000176

Ashton, M.C., Lee, K., \& de Vries, R.E. (2014). The HEXACO Honesty-Humility, Agreeableness, and Emotionality Factors. Personality and Social Psychology Review, 18(2), 139-152. https:// doi.org/10.1177/1088868314523838

Barelds, D.P.H. \& De Raad, B. (2015). The role of word-categories in trait-taxonomy: evidence from the Dutch personality taxonomy. International Journal of Personality Psychology, 1(1), 15-25. Retrieved from http://jdbsc.rug.nl/index.php/ijpp/article/viewFile/19316/16792

Borghuis, J., Denissen, J.J.A., Oberski, D., Sijtsma, K., Meeus, W.H.J., Branje, S., \& Bleidorn, W. (2017). Big Five personality stability, change, and codevelopment across adolescence and early adulthood. Journal of Personality and Social Psychology. http://dx.doi.org/10.1037/ pspp0000138

Cattell, R.B. (1995). The fallacy of five factors in the personality sphere. The Psychologist, 8(5), 207-208.

Chandler, J. Likeableness and meaningfulness ratings of $555(+487)$ person-descriptive words. Journal of Research in Personality, 72, 50-57. https://doi.org/10.1016/j.jrp.2016.07.005

Davies, S.E., Connelly, B.S., Ones, D.S., \& Birkland, A.S. (2015). The General Factor of Personality: The "Big One," a self-evaluative trait, or a methodological gnat that won't go away? Personality and Individual Differences, 81, 13-22. https://doi.org/10.1016/j.paid.2015.01.006

De Raad, B. \& Mlačić, B. (2017). The Lexical Foundation of the Big Five-Factor Model. In T.A. Widiger (ed.). The Oxford Handbook of The Five Factor Model (191-216). Oxford University Press. https://doi.org/10.1093/oxfordhb/9780199352487.013.12 
de Vries, R.E. (2011). No evidence for a General Factor of Personality in the HEXACO Personality Inventory. Journal of Research in Personality, 45(2). https://doi.org/10.1016/j. jrp.2010.12.002

Eysenck, H.J. (1992). Four ways five factors are not basic. Personality and Individual Differencesvidual Differences, 13(6), 667-673. Retrieved from http://differentialclub.wdfiles.com/local-files/personality-structure/Eysenck1992_4ways_are_NOT_basic.pdf

Fan, Y., Zhichen, X., Beibei, C., \& Jixia, W. (2015). The characteristics of Chinese people's honesty-humility personality and its implicit and explicit relationships. Journal of Psychological Science, 5, 1162-1169.

Galton, F. (1884). Measurement of character. Fortnightly Review, 36, 179-185.

Gill, C.M. \& Hodgkinson, G.P. (2007). Development and validation of the Five-Factor Model Questionnaire (FFMQ): an adjectival-based personality inventory for use in occupational settings. Personnel Psychology, 60(3), 731-766. Retrieved from https://search.proquest.com/ docview/220134687?pq-origsite $=$ gscholar

Gurven, M., von Rueden, C., Massenkoff, M., Kaplan, H., \& Lero Vie, M. (2013). How universal is the Big Five? Testing the five-factor model of personality variation among forager-farmers in the Bolivian Amazon. Journal of Personality and Social Psychology, 104(2), 354-70. https://doi.org/10.1037/a0030841

Han, M.-P., Seok, B.-I., \& Kim, J.-H. (2017). Effects of six personality factors of CEOs at small and medium-sized enterprises on performance in business management: Focusing on learning and growth. Asian Academy of Management Journal, 22(2), 97-128. https://doi. org/10.21315/aamj2017.22.2.4

Hodgkinson, G.P. \& Gill, C.M.H.D. (2015). Five Factor Model of Personalty. In C. Cooper (ed.), Wiley Encyclopedia of Management (3rd ed.). John Wiley \& Sons.

John, O.P., Angleitner, A., \& Ostendorf, F. (1988). The lexical approach to personality: a historical review of trait taxonomic research. European Journal of Personality, 2(3), 171-203. https://doi.org/10.1002/per.2410020302

Kajonius, P., Mac Giolla, E., Mai, N., French, B., Katigbak, M., \& Ortiz, F. (2017). Personality traits across countries: Support for similarities rather than differences. PLOS ONE, 12(6), e0179646. https://doi.org/10.1371/journal.pone.0179646

Kawamoto, T. (2016). Cross-sectional age differences in the HEXACO personality: Results from a Japanese sample. Journal of Research in Personality, 62, 1-5.

Leontiev, A.A. (2017). Osnovy Psikholingvistiki (Foundation of Psycholinguistics). Moscow: Litres.

Leutner, F., Ahmetoglu, G., Akhtar, R., \& Chamorro-Premuzic, T. (2014). The relationship between the entrepreneurial personality and the Big Five personality traits. Personality and individual differences, 63, 58-63. https://doi.org/10.1016/j.paid.2014.01.042

Litvinova, T. \& Ryzhkova, E. (2018). RusNeuroPsych: Open Corpus for Study Relations between Author Demographic, Personality Traits, Lateral Preferences and Affect in Text. International Journal of Open Information Technologies, 6(3), 32-36.

Loehlin, J.C. (2013). The general factor of personality: What lies beyond?-II. Personality and Individual Differences, 54(1). https://doi.org/10.1016/j.paid.2012.08.006

Mitchell, R.L.C. \& Kumari, V. (2016). Hans Eysenck's interface between the brain and personality: Modern evidence on the cognitive neuroscience of personality. Personality and Individual Differences, 103, 74-81. https://doi.org/10.1016/j.paid.2016.04.009

Mõttus, R., Kandler, C., Bleidorn, W., Riemann, R., \& McCrae, R.R. (2017). Personality traits below facets: The consensual validity, longitudinal stability, heritability, and utility of personality nuances. Journal of Personality and Social Psychology, 112(3), 474-490. https://doi. org/10.1037/pspp0000100 
Power, R.A. \& Pluess, M. (2015). Heritability estimates of the Big Five personality traits based on common genetic variants. Translational psychiatry, 5(7), e604. doi: 10.1038/tp.2015.96

Putilov, A.A. (2018). A 3-D Look at the Russian Personality Traits Structure. Current Psychology, 37(3), 528-542. https://doi.org/10.1007/s12144-016-9535-y

Saucier, G. \& Goldberg, L.R. (1996). Evidence for the Big Five in analyses of familiar English personality adjectives. European Journal of Personality, 10(1), 61-77. https://doi.org/10.1002/ (SICI) 1099-0984(199603)10:1<61::AID-PER246>3.0.CO;2-D

Schmitt, D.P., Allik, J., Mccrae, R.R., Alcalay, L., Bennett, K.L., Bianchi, G., ... \& Panayiotou, A. (2007). The Geographic Distribution of Big Five Personality Traits: Patterns and Profiles of Human Self-Description across 56 Nations. Journal of Cross-Cultural Psychology, 38(2), 173-212. https://doi.org/10.1177/0022022106297299

Shmelev, A.G. (2002). Psikhodiagnostika lichnostnykh chert (Psychodyagnostics of personality traits). Saint-Petersburg: Rech.

Shu, F., McAbee, S.T., \& Ayman, R. (2017). The HEXACO personality traits, cultural intelligence, and international student adjustment. Personality and Individual Differences, 106, 21-25. http://dx.doi.org/10.1016/j.paid.2016.10.024

Strouts, P.H., Brase, G.L., \& Dillon, H.M. (2017). Personality and evolutionary strategies: The relationships between HEXACO traits, mate value, life history strategy, and sociosexuality. Personality and Individual Differences, 115, 128-132. https://doi.org/10.1016/j. paid.2016.03.047

Trofimova, I. (2016). The interlocking between functional aspects of activities and a neurochemical model of adult temperament in M. C. Arnold (ed), Temperaments: individual differences, social and environmental influences and impact on quality of life, 77-147. Nova Science Publishers: Haupaugge, New York.

Uher, J. (2013). Personality Psychology: Lexical Approaches, Assessment Methods, and Trait Concepts Reveal Only Half of the Story - Why it is Time for a Paradigm Shift. Integrated Psychological Behavior, 47, 1-55. https://doi.org/10.1007/s12124-013-9230-6

Velichkovsky, B.M. \& Rumbaugh, D.M. (2013). Communicating meaning: The evolution and development of language. Psychology Press.

Vygotsky, L.S. (1987). Thinking and speech. The collected works of L.S. Vygotsky, 1, 39-285.

Waller, V. (1995). The Big Seven Factor Model of Personality Description: Evidence for Its CrossCultural Generality in a Spanish Sample. Journal of Personality and Social Psychology, 69(4), 701-718. https://doi.org/10.1037/0022-3514.69.4.701

Wright, A.G.C. (2017). Factor analytic support for the five-factor model. In T.A. Widiger (ed.), The Oxford Handbook of the Five Factor Model ( pp. 217-242). Oxford University Press.

Original manuscript received October 01, 2018

Revised manuscript accepted January 10, 2019

First published online February 15, 2019

To cite this article: Gill, C.M.H.D., Berezina E. (2019). Modeling personality structure using semantic relationships: is the HEXACO honesty-humility a distinct trait? Psychology in Russia: State of the Art, 12(1), 89-103. DOI: 10.11621/pir.2019.0107 